\title{
Korean Shock Society septic shock registry: a preliminary report
}

\section{Tae Gun Shin' ${ }^{1}$, Sung Yeon Hwang', Gu Hyun Kang' ${ }^{2}$ Won Young Kim³, Seung Mok Ryoo ${ }^{3}$, Kyuseok Kim${ }^{4}$, You Hwan Jo ${ }^{4}$, Sung Phil Chung, Young Seon $\mathrm{Joo}^{5}$, Jin Ho Beom ${ }^{5}$, Sung-Hyuk Choi ${ }^{6}$, Young Hoon Yoon ${ }^{6}$, Woon Yong Kwon ${ }^{7}$, Tae Ho Lim ${ }^{8}$, Kap Su Han ${ }^{9}$, Han Sung Choi ${ }^{10}$, Gil Joon Suh ${ }^{7}$}

\begin{abstract}
Department of Emergency Medicine, ${ }^{1}$ Samsung Medical Center, Sungkyunkwan University School of Medicine, Seoul; ${ }^{2}$ Hallym University College of Medicine, Seoul; ${ }^{3}$ Asan Medical Center, University of Ulsan College of Medicine, Seoul; ${ }^{4}$ Seoul National University Bundang Hospital, Seongnam; ${ }^{5}$ Yonsei University College of Medicine, Seoul; ${ }^{6}$ Guro Hospital, Korea University Medical Center, Seoul; ${ }^{7}$ Seoul National University College of Medicine, Seoul; ${ }^{8}$ Hanyang University College of Medicine, Seoul; ${ }^{9}$ Korea University Anam Hospital, Seoul; ${ }^{10}$ Kyung Hee University Hospital, Seoul, Korea
\end{abstract}

Objective To evaluate the clinical characteristics, therapeutic interventions, and outcomes of patients with septic shock admitted to the emergency department (ED).

Methods This study was a preliminary, descriptive analysis of a prospective, multi-center, observational registry of the EDs of 10 hospitals participating in the Korean Shock Society. Patients aged 19 years or older who had a suspected or confirmed infection and evidence of refractory hypotension or hypoperfusion were included.

Results A total of 468 patients were enrolled (median age, 71.3 years; male, 55.1\%; refractory hypotension, 82.9\%; hyperlactatemia without hypotension, 17.1\%). Respiratory infection was the most common source of infection (31.0\%). The median Sepsis-related Organ Failure Assessment score was 7.5. The sepsis bundle compliance was $91.2 \%$ for lactate measurement, $70.3 \%$ for blood culture, $68.4 \%$ for antibiotic administration, $80.3 \%$ for fluid resuscitation, $97.8 \%$ for vasopressor application, $68.0 \%$ for central venous pressure measurement, $22.0 \%$ for central venous oxygen saturation measurement, and 59.2\% for repeated lactate measurement. Among patients who underwent interventions for source control $(n=117,25.1 \%), 43(36.8 \%)$ received interventions within 12 hours of ED arrival. The in-hospital, 28-day, and 90-day mortality rates were $22.9 \%, 21.8 \%$, and $27.1 \%$, respectively. The median ED and hospital lengths of stay were 6.8 hours and 12 days, respectively.

Conclusion This preliminary report revealed a mortality of over $20 \%$ in patients with septic shock, which suggests that there are areas for improvement in terms of the quality of initial resuscitation and outcomes of septic shock patients in the ED.

Keywords Sepsis; Septic shock; Mortality; Patient care bundles
Received: 18 July 2017

Revised: 20 August 2017

Accepted: 22 August 2017

Correspondence to: Gil Joon Suh Department of Emergency Medicine, Seoul National University College of Medicine, 101 Daehak-ro, Jongro-gu, Seoul 03080, Korea

E-mail: suhgil@snu.ac.kr

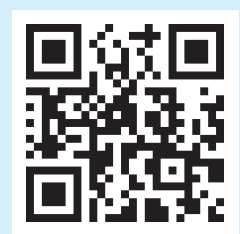

How to cite this article:

Shin TG, Hwang SY, Kang GH, Kim WY, Ryoo SM, Kim K, Jo YH, Chung SP, Joo YS, Bum JH, Choi SH, Yoon YH, Kwon WY, Lim TH, Han KS, Choi HS, Suh GJ. Korean Shock Society septic shock registry: a preliminary report. Clin Exp Emerg Med 2017;4(3):146-153.

This is an Open Access article distributed under the terms of the Creative Commons Attribution Non-Commercial License (http:// creativecommons.org/licenses/by-nc/4.0/). 


Capsule
Wummary is already known
$\begin{aligned} & \text { Mortality in patients with sepsis or septic shock remains high, while compliance with the sepsis bundle metrics remains } \\ & \text { low and variable. }\end{aligned}$
$\begin{aligned} & \text { What is new in the current study } \\ & \text { This is the first, multi-center study on septic shock in the emergency departments of Korea. The results of this prelimi- } \\ & \text { nary report suggest that initial sepsis care and the outcomes of septic shock patients need to be improved. }\end{aligned}$

\section{INTRODUCTION}

Shock refers to circulatory failure with inadequate tissue perfusion and cellular oxygen utilization.' Sepsis is the most common cause of noncardiogenic shock, which is accompanied by lifethreatening organ dysfunction due to dysregulated host response to infection..$^{1-3}$ Despite current advances in intensive care and sepsis therapy, the incidence of sepsis continues to rise and mortality rates in patients with septic shock exceed $20 \%{ }^{4,5}$ With no specific superior therapeutic options being developed, further research and clinical quality improvement are needed to increase the survival of patients with septic shock. ${ }^{6,7}$

The Korean Shock Society (KoSS) was established in 2013 to research and improve clinical quality in the diagnosis and treatment of shock, which is a principal topic in emergency and critical care medicine. The KoSS has been prospectively collecting data in a septic shock registry since October 2015 as a first step towards future research on shock. The KoSS septic shock registry data include clinical characteristics and initial management of, and early outcomes in patients presenting to emergency departments (EDs) with septic shock. This study is a preliminary, descriptive analysis of the KoSS septic shock registry.

\section{METHODS}

\section{Study design}

This prospective, multi-center, observational study was designed to evaluate the clinical characteristics, therapeutic interventions, and outcomes of patients with septic shock in EDs. Among hospitals in the KoSS, 10 EDs participated in the KoSS septic shock registry from October 2015 to June 2016 (Table 1). Patients aged 19 years or older who visited one of these EDs and met the eligibility criteria were enrolled. The inclusion criteria included suspected or confirmed infection and evidence of refractory hypotension or hypoperfusion. ${ }^{8-10}$ Systemic inflammatory response
Table 1. Participating hospitals

\begin{tabular}{lcl}
\hline Hospital & No. of patients (\%) & \multicolumn{1}{c}{ Enrollment period } \\
\hline A & $78(16.7)$ & December 2015 to April $2016(5 \mathrm{mo})$ \\
B & $39(8.3)$ & November 2015 to April $2016(6 \mathrm{mo})$ \\
C & $31(6.6)$ & March 2016 to April $2016(2 \mathrm{mo})$ \\
D & $14(3.0)$ & December 2015 to April $2016(5 \mathrm{mo})$ \\
E & $57(12.2)$ & November 2015 to June $2016(8 \mathrm{mo})$ \\
F & $103(22.0)$ & November 2015 to June $2016(8 \mathrm{mo})$ \\
G & $27(5.8)$ & January 2016 to June $2016(6 \mathrm{mo})$ \\
H & $66(14.1)$ & January 2016 to June $2016(6 \mathrm{mo})$ \\
I & $32(6.8)$ & November 2015 to January 2016 (3 mo) \\
J & $21(4.5)$ & January 2016 to April $2016(4 \mathrm{mo})$ \\
\hline
\end{tabular}

syndrome criteria were not obligatory for enrollment." Hypotension was defined as a systolic blood pressure $<90 \mathrm{mmHg}$, a mean arterial pressure $<70 \mathrm{mmHg}$, or a systolic blood pressure decrease $>40 \mathrm{mmHg}{ }^{6}$ Refractory hypotension was defined as persistent hypotension after intravenous fluid challenge ( $1 \mathrm{~L}$ or more administered over 30 minutes) or as the need for vasopressors after fluid resuscitation. Hypoperfusion was defined as a serum lactate concentration of $4 \mathrm{mmol} / \mathrm{L}$ or greater. Patients who signed a "Do not attempt resuscitation" order before ED arrival or at the time of diagnosis, met the inclusion criteria 6 hours after ED arrival, were transferred from other hospitals without meeting the inclusion criteria upon ED arrival, or were directly transferred from EDs to other hospitals were excluded from this study. The study was approved by the institutional review boards of the individual participating centers and informed consent was obtained according to local institutional review board policy.

\section{Data collection}

All data were anonymized and collected using standardized case report forms by research coordinators or physicians at each participating hospital. For data quality control, data were centrally reviewed at the coordinating hospital and case report instructions were provided via manuals and training meetings. The following 
data were included in the registry: demographic characteristics, comorbidities, vital signs, suspected infection source, and laboratory data. We investigated interventions including the Surviving Sepsis Campaign (SSC) bundle: lactate measurement, blood culture, fluid resuscitation, central venous pressure (CVP) measurement, central venous oxygen saturation $\left(\mathrm{ScvO}_{2}\right)$ measurement, vasopressor use, transfusion, mechanical ventilation, renal replacement therapy, and interventions for infection source control. ${ }^{6}$ The outcome variables included in-hospital, 28-day, and 90day mortality; ED disposition; and length of stay in the intensive care unit and hospital. Maximum sepsis-related Organ Failure Assessment (SOFA) and Acute Physiology and Chronic Health evaluation (APACHE) II scores were evaluated using the worst parameters within 24 hours after ED arrival. ${ }^{12,13}$

\section{Statistical analyses}

The results are presented as medians with interquartile ranges (IORs) for continuous variables and as the numbers of patients and percentages for categorical data. Continuous and categorical variables were analyzed using Wilcoxon rank-sum and chi-square

Table 2. Baseline characteristics

\begin{tabular}{|c|c|c|c|c|}
\hline Variable & Overall $(n=468)$ & Survivor $(n=361)$ & Non-survivor $(n=107)$ & P-value \\
\hline Age (yr) & $71.3(59.9-78.9)$ & $71.3(58.7-78.4)$ & $72.2(61.8-80.3)$ & 0.15 \\
\hline Sex (male) & $258(55.1)$ & $186(51.5)$ & $72(67.3)$ & $<0.01$ \\
\hline \multicolumn{5}{|l|}{ Initial presentation } \\
\hline Refractory hypotension & $388(82.9)$ & $312(86.4)$ & 76 (71.0) & $<0.01$ \\
\hline Hyperlactatemia without hypotension & $80(17.1)$ & $49(13.6)$ & $31(29.0)$ & $<0.01$ \\
\hline \multicolumn{5}{|l|}{ Comorbidities } \\
\hline Hypertension & $214(45.7)$ & $167(46.3)$ & $47(43.9)$ & 0.67 \\
\hline Diabetes & $142(30.3)$ & $102(28.3)$ & $40(37.4)$ & 0.07 \\
\hline Cardiac disease & 65 (13.9) & 49 (13.6) & $16(15.0)$ & 0.71 \\
\hline Cerebrovascular disease & $77(16.5)$ & $61(16.9)$ & $16(15.0)$ & 0.63 \\
\hline Chronic lung disease & $31(6.6)$ & $22(6.1)$ & $9(8.4)$ & 0.40 \\
\hline Chronic renal disease & $51(10.9)$ & $33(9.1)$ & $18(16.8)$ & 0.03 \\
\hline Liver cirrhosis & $44(9.4)$ & $36(10.0)$ & $8(7.5)$ & 0.44 \\
\hline Metastatic solid cancer & $82(17.5)$ & $59(16.3)$ & $23(21.5)$ & 0.22 \\
\hline Hematologic malignancy & $23(4.9)$ & $11(3.1)$ & $12(11.2)$ & $<0.01$ \\
\hline Transplant & $4(0.9)$ & $3(0.8)$ & $1(0.9)$ & 0.92 \\
\hline Dementia & $38(8.1)$ & $29(8.0)$ & $9(8.4)$ & 0.90 \\
\hline Transfer from another hospital & $169(36.1)$ & $130(36.0)$ & $39(36.5)$ & 0.93 \\
\hline Referred from long-term care facilities/hospitals & $51(10.9)$ & $39(10.8)$ & $12(11.2)$ & 0.90 \\
\hline \multicolumn{5}{|l|}{ Suspected infection focus } \\
\hline Respiratory infection & $145(31.0)$ & $92(25.5)$ & $53(49.5)$ & $<0.01$ \\
\hline Intra-abdominal infection & $115(24.6)$ & $98(27.1)$ & $17(15.9)$ & $<0.01$ \\
\hline Urinary tract infection & $99(21.1)$ & $86(23.8)$ & $13(12.2)$ & $<0.01$ \\
\hline Mixed & $60(12.8)$ & 45 (12.5) & $15(14.0)$ & $<0.01$ \\
\hline Other or unknown & $49(10.5)$ & $40(11.1)$ & $9(8.4)$ & $<0.01$ \\
\hline \multicolumn{5}{|l|}{ Initial vital signs } \\
\hline Systolic blood pressure (mmHg) & $95(80-122)$ & $96(80-120)$ & $91(79-131)$ & 0.72 \\
\hline Respiratory rate (/min) & $20(20-24)$ & $20(20-24)$ & 22 (19-28) & 0.01 \\
\hline Heart rate (/min) & $106(91-125)$ & $104(90-124)$ & $112(96-128)$ & 0.02 \\
\hline Body temperature $\left({ }^{\circ} \mathrm{C}\right)$ & $37.4(36.5-38.4)$ & $37.6(36.6-38.5)$ & $37.0(36.2-37.8)$ & $<0.01$ \\
\hline \multicolumn{5}{|l|}{ Vital signs at enrollment } \\
\hline Systolic blood pressure $(\mathrm{mmHg})$ & $84(74-94)$ & $84(74-94)$ & $82(72-96)$ & 0.58 \\
\hline Respiratory rate (/min) & $20(18-24)$ & $20(18-24)$ & $21(20-26)$ & 0.01 \\
\hline Heart rate $(/ \mathrm{min})$ & $102(89-119)$ & $100(88-118)$ & $108(95-122)$ & $<0.01$ \\
\hline Body temperature $\left({ }^{\circ} \mathrm{C}\right)$ & $37.2(36.5-38.0)$ & $37.4(36.6-38.2)$ & $36.8(36.3-37.6)$ & $<0.01$ \\
\hline \multicolumn{5}{|l|}{ Culture positive } \\
\hline Blood & $198(42.4)$ & $158(43.8)$ & $40(37.4)$ & 0.37 \\
\hline Other & $247(52.8)$ & $183(50.7)$ & $64(59.8)$ & 0.10 \\
\hline Resistant to initial antibiotics & $48(10.3)$ & $36(10.0)$ & $12(11.3)$ & 0.71 \\
\hline Maximum SOFA score in 24 hours & $7.5(5-10.5)$ & $7(5-10)$ & $9(7-12)$ & $<0.01$ \\
\hline APACHE II score & $18.5(13-25)$ & 17 (12-24) & 22 (18-29) & $<0.01$ \\
\hline
\end{tabular}

Data are shown as median (interquartile range) or number (\%).

SOFA, Sepsis-related Organ Failure Assessment; APACHE, Acute Physiology and Chronic Health Evaluation. 
tests, respectively. P-values less than 0.05 were considered statistically significant. STATA ver. 13.0 (STATA Corp., College Station, TX, USA) was used for statistical analysis.

\section{RESULTS}

\section{Baseline characteristics and laboratory data}

A total of 468 patients with suspected septic shock were enrolled. We excluded patients with "Do not attempt resuscitation" orders $(n=60)$, those who met the inclusion criteria six hours after ED arrival $(n=44)$, those who were directly transferred from EDs to other hospitals ( $n=32)$, those who were transferred from other hospitals without meeting the inclusion criteria upon ED arrival $(n=13)$, and those who had not provided informed consent $(n=460)$.

The baseline characteristics and comparisons between survivors and non-survivors according to hospital mortality are shown in Table 2. The median age was 71.3 years (IOR, 59.9 to 78.9) and
$258(55.1 \%)$ patients were male. Of the enrolled patients, 388 $(82.9 \%)$ had refractory hypotension and $80(17.1 \%)$ had hyperlactatemia without hypotension on initial presentation. Respiratory infections (31.0\%), intra-abdominal infections (24.6\%), and urinary tract infections $(21.1 \%)$ were the most common sources of infection. Bacteremia was present in 198 (42.4\%) patients. The median maximum SOFA score was $7.5(I Q R, 5$ to 10.5) and median APACHE II score was 18.5 (IQR, 13 to 25). Between survivors and non-survivors, there were significant differences in variables including sex, initial presentation, chronic renal disease, hematologic malignancy, suspected infection focus, respiratory rate, heart rate, body temperature, SOFA score, and APACHE II score.

The laboratory results are shown in Table 3, including complete blood count, blood chemistry, and lactate level. The median lactate level was $3.1 \mathrm{mmol} / \mathrm{L}(\mathrm{IQR}, 1.8$ to 5.0$)$ on initial measurement ( $\mathrm{n}=458)$ and $2.5 \mathrm{mmol} / \mathrm{L}(\mathrm{IQR}, 1.6$ to 4.5$)$ at the second measurement $(n=353)$.

Table 3. Laboratory test findings

\begin{tabular}{|c|c|c|c|c|c|}
\hline Variable & Overall $(n=468)$ & Survivor $(n=361)$ & Non-survivor $(n=107)$ & P-value & No. of measurements \\
\hline Initial lactate (mmol/L) & $3.1(1.8-5.0)$ & $2.9(1.8-4.7)$ & $4.4(2.5-7.5)$ & $<0.01$ & 458 \\
\hline Second lactate $(\mathrm{mmol} / \mathrm{L})$ & $2.5(1.6-4.5)$ & $2.3(1.5-3.9)$ & $3.4(1.9-6.8)$ & $<0.01$ & 353 \\
\hline White blood cell count $\left(\times 10^{3} / \mathrm{L}\right)$ & $11.60(6.34-18.35)$ & $12.06(7.00-19.30)$ & $8.58(3.50-14.90)$ & $<0.01$ & 468 \\
\hline Hemoglobin $(\mathrm{g} / \mathrm{dL})$ & $11.1(9.4-12.6)$ & $11.3(9.7-12.7)$ & $10.3(8.6-11.9)$ & $<0.01$ & 468 \\
\hline Hematocrit (\%) & $33.7(28.9-38.0)$ & $34.2(29.9-38.3)$ & $31.5(26.4-36.9)$ & 0.01 & 468 \\
\hline Platelet count $\left(\times 10^{3} / \mathrm{L}\right)$ & $154(89-244)$ & $162(95-246)$ & $124(57-221)$ & 0.03 & 468 \\
\hline Sodium (mmol/L) & $135(131-138)$ & $135(132-138)$ & $135(131-139)$ & 0.81 & 468 \\
\hline Potassium (mmol/L) & $4.1(3.6-4.6)$ & $4.0(3.6-4.5)$ & $4.3(3.7-4.8)$ & 0.01 & 468 \\
\hline Chloride (mmol/L) & $100(96-105)$ & $100(97-105)$ & 99 (94-105) & 0.13 & 467 \\
\hline Blood urea nitrogen (mg/dL) & $27.0(18.9-43.4)$ & $26.0(18.0-40.0)$ & $32.1(21.0-49.0)$ & $<0.01$ & 466 \\
\hline Creatinine $(\mathrm{mg} / \mathrm{dL})$ & $1.37(0.91-2.27)$ & $1.31(0.90-2.15)$ & $1.70(1.01-2.67)$ & 0.02 & 466 \\
\hline AST (U/L) & $40(25-85)$ & $39(25-80)$ & $41(27-116)$ & 0.17 & 467 \\
\hline ALT (U/L) & $26(14-56)$ & $27(14-59)$ & $24(13-43)$ & 0.35 & 467 \\
\hline Albumin (g/dL) & $3.0(2.6-3.4)$ & $3.1(2.6-3.5)$ & $2.8(2.3-3.2)$ & $<0.01$ & 455 \\
\hline Prothrombin time (INR) & $1.27(1.12-1.46)$ & $1.23(1.11-1.42)$ & $1.32(1.20-1.55)$ & $<0.01$ & 455 \\
\hline C-reactive protein (mg/dL) & $14.47(5.91-23.44)$ & $14.27(5.57-23.01)$ & $15.43(7.28-26.38)$ & 0.17 & 468 \\
\hline Glucose (mg/dL) & $136(106-190)$ & $136(105-187)$ & 137 (109-212) & 0.52 & 467 \\
\hline Arterial pH & $7.43(7.36-7.47)$ & $7.43(7.38-7.48)$ & $7.38(7.26-7.46)$ & $<0.01$ & 461 \\
\hline $\mathrm{PaCO}_{2}(\mathrm{mmHg})$ & $29.0(24.7-34.0)$ & $29.0(25.0-33.7)$ & $29.0(23.5-37.9)$ & 0.85 & 461 \\
\hline $\mathrm{PaO}_{2}(\mathrm{mmHg})$ & $77.1(62.3-95.7)$ & $79.0(63.8-95.7)$ & $72.8(57.0-96.0)$ & 0.11 & 461 \\
\hline Bicarbonate (arterial, $\mathrm{mmol} / \mathrm{L}$ ) & $19.0(15.6-22.4)$ & $19.5(16.4-22.6)$ & $17.7(12.8-21.2)$ & $<0.01$ & 461 \\
\hline Initial ScvO $\mathrm{O}_{2}(\%)$ & $69.4(59.0-77.9)$ & $69.2(56.0-80.1)$ & 70.7 (59.0-76.8) & 0.70 & 121 \\
\hline Procalcitonin (mmol/L) & $6.73(0.96-26.80)$ & $6.37(0.98-26.66)$ & $7.75(0.78-30.56)$ & 0.50 & 389 \\
\hline D-dimer (mcg/mL) & $3.67(2.12-8.87)$ & $3.52(2.04-7.84)$ & $4.20(2.64-11.30)$ & 0.10 & 295 \\
\hline Troponin I or T (ng/mL) & $0.047(0.020-0.179)$ & $0.041(0.018-0.146)$ & $0.075(0.022-0.300)$ & 0.06 & 384 \\
\hline NT-proBNP (pg/mL) & $2,426(928-6,808)$ & $2,499(877-6,881)$ & $2,335(1,032-6,734)$ & 0.90 & 116 \\
\hline BNP $(p g / m L)$ & 195 (79-568) & 135 (70-429) & 303 (131-949) & $<0.01$ & 145 \\
\hline
\end{tabular}

Data are shown as median (interquartile range).

AST, aspartate transaminase; $\mathrm{ALT}$, alanine transaminase; INR, international normalized ratio; $\mathrm{ScvO}_{2}$, central venous oxygen saturation; NT-proBNP, N-terminal pro-brain natriuretic peptide; BNP, brain natriuretic peptide. 


\section{Interventions}

The interventions or procedures for the diagnosis and management of septic shock are shown in Table 4. The median time from
ED arrival was $0.4(I Q R, 0.2$ to 0.8$)$ hours for lactate measurement, 1.6 (IQR, 0.5 to 3.6) hours for blood culture, and 2.2 (IQR, 1.3 to 3.3 ) hours for first antibiotic administration. Within the

Table 4. Interventions

\begin{tabular}{|c|c|c|c|c|}
\hline Variable & Overall $(n=468)$ & Survivor $(n=361)$ & Non-survivor $(n=107)$ & P-value \\
\hline Lactate measurement & 458 (97.9) & $354(98.1)$ & $104(97.2)$ & 0.59 \\
\hline Time from ED arrival to initial Lactate Measurement (hr) & $0.4(0.2-0.8)$ & $0.4(0.2-0.9)$ & $0.3(0.1-0.7)$ & $<0.01$ \\
\hline Measured within 3 hours ${ }^{b)}$ & $427(91.2)$ & $329(91.1)$ & $98(91.6)$ & 0.88 \\
\hline Blood culture & $464(99.4)$ & $358(99.2)$ & $107(100)$ & 0.34 \\
\hline Time from ED arrival to blood culture (hr) & $1.6(0.5-3.6)$ & $1.5(0.5-3.0)$ & $2.2(0.5-7.0)$ & 0.02 \\
\hline Blood culture within 3 hours ${ }^{\text {b) }}$ & $329(70.3)$ & $270(74.8)$ & $59(55.1)$ & $<0.01$ \\
\hline Time from ED arrival to first antibiotic administration $(\mathrm{hr})^{\mathrm{a})}$ & $2.2(1.3-3.3)$ & $2.1(1.3-3.2)$ & $2.5(1.5-3.4)$ & 0.2 \\
\hline Administered within 3 hours $^{b)}$ & $320(68.4)$ & $254(70.4)$ & $66(61.7)$ & 0.09 \\
\hline \multicolumn{5}{|l|}{ Fluid resuscitation } \\
\hline Fluid administration (30 mL/kg of crystalloid) within 3 hours ${ }^{\mathrm{b}, \mathrm{c}}$ ) & $376(80.3)$ & $289(80.1)$ & $87(81.3)$ & 0.78 \\
\hline \multicolumn{5}{|l|}{ Fluids for initial resuscitation } \\
\hline Normal saline & $387(82.7)$ & $297(82.3)$ & $90(84.1)$ & 0.66 \\
\hline Synthetic colloids & $45(9.6)$ & $27(7.5)$ & 18 (16.8) & $<0.01$ \\
\hline Central venous catheter insertion within 6 hours $^{\mathrm{c}, \mathrm{d})}$ & $318(68.0)$ & $240(66.5)$ & $78(72.9)$ & 0.21 \\
\hline CVP measurement within 6 hours ${ }^{\mathrm{b}, \mathrm{d})}$ & $253(54.1)$ & $193(53.5)$ & $60(56.1)$ & 0.63 \\
\hline $\mathrm{ScvO}_{2}$ measurement within 6 hours $^{\mathrm{b}, \mathrm{d})}$ & $103(22.0)$ & $76(21.1)$ & $27(25.2)$ & 0.36 \\
\hline Application of vasopressors for achievement MAP $\geq 65 \mathrm{mmHg}^{\mathrm{b}, e)}$ & $458(97.9)$ & $354(98.1)$ & 104 (97.2) & 0.59 \\
\hline \multicolumn{5}{|l|}{ Vasopressors } \\
\hline Time from ED arrival to first vasopressor administration (hr) & $1.9(1.0-3.5)$ & $2.0(1.2-3.5)$ & $1.4(0.7-2.4)$ & $<0.01$ \\
\hline First vasopressor & $431(92.1)$ & $333(92.2)$ & $98(91.6)$ & 0.83 \\
\hline Norepinephrine & $413(88.2)$ & $320(88.6)$ & $93(86.9)$ & 0.63 \\
\hline Dopamine & $17(3.6)$ & $12(3.3)$ & $5(4.7)$ & 0.51 \\
\hline Other & $1(0.2)$ & $1(0.3)$ & $0(0)$ & 0.59 \\
\hline Second vasopressor & 79 (16.9) & $46(12.7)$ & $33(30.8)$ & $<0.01$ \\
\hline Red blood cells & $70(15.0)$ & 39 (10.8) & $31(29.0)$ & $<0.01$ \\
\hline Fresh frozen plasma & $44(9.4)$ & $18(5.0)$ & $26(24.3)$ & $<0.01$ \\
\hline Platelets & $52(11.1)$ & $31(8.6)$ & $21(19.6)$ & $<0.01$ \\
\hline Mechanical ventilation & $151(32.3)$ & $76(21.1)$ & $76(71.0)$ & $<0.01$ \\
\hline Time to mechanical ventilation (hr) ${ }^{\text {a) }}$ & $2.4(0.6-9.4)$ & $2.3(0.6-7.0)$ & $2.5(0.6-11.0)$ & 0.93 \\
\hline Duration of mechanical ventilation (day) $)^{a}$ & $4(2-9)$ & $5(2-9)$ & $3(1.5-9)$ & 0.21 \\
\hline Renal replacement therapy & $73(15.6)$ & $25(6.9)$ & $48(44.9)$ & $<0.01$ \\
\hline Intervention for source control & $117(25.1)$ & $99(26.4)$ & $18(16.8)$ & 0.03 \\
\hline Time from ED arrival to initial source contro|a) & $15.6(7.2-47.9)$ & $15.2(7.2-36.0)$ & $20.9(7.1-96.1)$ & 0.59 \\
\hline Intervention within 12 hours from ED arrival & $43(9.2)$ & $38(10.5)$ & $5(4.7)$ & 0.07 \\
\hline
\end{tabular}

Data are shown as number (\%) or median (interquartile range).

$E D$, emergency department; $C V P$, central venous pressure; $\mathrm{ScvO}_{2}$, central venous oxygen saturation; $M A P$, mean arterial pressure.

${ }^{a}$ Missing data: one case, time to lactate measurement; five cases, time to antibiotics; six cases, time to vasopressors; 11 cases, time to mechanical ventilation; three cases, duration of mechanical ventilation; and seven cases, time to source control. ${ }^{b}$ The sepsis bundle components. ${ }^{\text {'T }}$ wo cases with central venous catheter insertion at other hospitals were not included. ${ }^{d}$ Time zero was considered as the time of hypotension or lactate elevation $\geq 4 \mathrm{mmol} / \mathrm{L}$. ${ }^{\mathrm{e}}$ Cases without hypotension were considered as the bundle component was achieved. 
initial three hours of ED arrival, lactate measurement was performed in 427 (91.2\%) patients, blood culture was done in 329 (70.3\%) patients, and initial antibiotics were administered to 320 (68.4\%) patients. Repeated lactate measurements were performed in 277 (59.2\%) patients within six hours of the first measurement. Initial fluid resuscitation (more than $30 \mathrm{~mL} / \mathrm{kg}$ of crystalloid) was performed in 376 (80.3\%) patients, most often using normal saline (82.7\%). CVP and $\mathrm{ScvO}_{2}$ were measured in 318 $(68.0 \%)$ and $103(22.0 \%)$ patients within six hours of the time of hypotension or lactate elevation, respectively. The compliance with the application of vasopressors for achievement of mean arterial pressure $\geq 65 \mathrm{mmHg}$ was $97.9 \%$. The median time from ED arrival to first vasopressor administration, most commonly norepinephrine, was 1.9 (IOR, 1.0 to 3.5) hours. Mechanical ventilation was applied in $151(32.3 \%)$ patients and renal replacement therapy was used in $73(15.6 \%)$ patients. The median time from ED arrival to first source control was 15.6 (IQR, 7.2 to 47.9$)$ hours. Among patients who underwent intervention for source control, 43 (36.8\%) received intervention within 12 hours of their ED arrival. Between survivors and non-survivors, there were significant differences in interventions, including time to lactate measurement, time to blood culture, use of synthetic colloids, time to the first vasopressor, use of a second vasopressor, transfusion, mechanical ventilation, and renal replacement therapy.

\section{Outcomes}

The in-hospital, 28-day, and 90-day mortality rates were $22.9 \%$, $21.8 \%$, and $27.1 \%$, respectively (Table 5 ). The intensive care unit admission rate from the ED was $64.5 \%$. The median length of

Table 5. Outcomes and lengths of stay

\begin{tabular}{lc}
\hline Variable & Value \\
\hline Hospital dischargea) & \\
Discharge & $305(65.1)$ \\
Transfer to another hospital & $56(12.0)$ \\
In-hospital mortality & $107(22.9)$ \\
28-Day mortality & $102(21.8)$ \\
Loss to follow-up & $20(4.3)$ \\
90-Day mortality & $127(27.1)$ \\
Loss to follow-up & $68(14.5)$ \\
Emergency department disposition & \\
Intensive care unit & $302(64.5)$ \\
General ward & $163(34.8)$ \\
Discharge & $2(0.4)$ \\
Death & $1(0.2)$ \\
Length of stay & \\
Emergency department (hr) & $6.8(4.0-14.6)$ \\
Intensive care unit (day) & $4(2-8)$ \\
Hospital (day) & $12(7-21)$ \\
\hline
\end{tabular}

Data are shown as number (\%) or median (interquartile range).

${ }^{a)}$ Eight cases with withdrawal of supportive care were included. stay in the ED, intensive care unit, and hospital ward were 6.8 (IQR, 4.0 to 14.6) hours, 4 (IQR, 2 to 8) days, and 12 (IQR, 7 to 21) days, respectively.

\section{DISCUSSION}

This preliminary analysis of a prospective, observational registry of 10 EDs participating in the KoSS is the first multi-center study about septic shock in EDs of Korea. The study mainly investigated the demographic characteristics, interventions, and clinical outcomes of patients with septic shock presenting with refractory hypotension or hyperlactatemia.

Mortality rates vary according to inclusion criteria and septic shock definition. ${ }^{14}$ Overall, the mortality trend in sepsis or septic shock patients is declining, but mortality remains high. ${ }^{4,5,15}$ Recent large clinical trials (the Australasian Resuscitation in Sepsis Evaluation study and the Protocolised Management in Sepsis trial), multi-center, ED-based studies for patients with septic shock, have reported $15.0 \%$ to $25.1 \%$ in-hospital mortality and $15.3 \%$ to $24.6 \% 28$-day mortality. ${ }^{8} 10$ Our study, with similar inclusion criteria to these trials, showed comparable mortality rates.

According to a global observation study (the International Multicentre Prevalence Study on Sepsis, including 1,794 patients from 62 countries), compliance with the sepsis bundle metrics remains low and variable. ${ }^{16}$ Compliance rates were comparable in interventions including blood culture, antibiotic administration, application of vasopressor, CVP measurement, and repeated lactate measurement. However, higher compliance rates were observed for initial lactate measurement and initial fluid resuscitation. $\mathrm{ScvO}_{2}$ was relatively underused; the practice was probably affected by the current trials for early goal-directed therapy. ${ }^{8-10}$

There are some controversies regarding whether sepsis bundle compliance directly improves outcomes and whether specific interventions or protocols are superior to management by clinical assessment. ${ }^{3}$ However, considering the clinical importance of early recognition and timely management of septic shock, compliance to the sepsis bundle might be associated with clinical improvement in the quality of initial treatment and better outcomes. ${ }^{17,18}$ From this perspective, we observed that some interventions for sepsis care might need to be improved, including obtaining a blood culture, antibiotic administration, and source control interventions.

Our inclusion criteria varied from the new definition of septic shock from Sepsis-3. ${ }^{19}$ However, the new definition is relatively specific and difficult to apply in the ED. Refractory hypotension and hyperlactatemia are criteria currently recommended by the SSC guidelines for the initiation of aggressive management. ${ }^{6}$ In 
addition, we have been collecting data about the Sepsis-3 definition and future studies from the KoSS registry will include these results.

There are some changes in the updated 2016 SSC guidelines. ${ }^{20}$ CVP and $\mathrm{ScVO}_{2}$ are no longer recommended as the initial goals of resuscitation. However, the use of these targets may be considered because current trials including less severely ill patients have reported that no harm was associated with the interventional strategies. ${ }^{8-10,20}$ The guidelines also recommend frequent reassessment of hemodynamic status using available physiologic variables and that dynamic rather than static variables be used to predict fluid responsiveness. The KoSS registry needs to reflect these recent changes.

This study has several limitations. Further data collection and analysis are needed because this is a preliminary, descriptive study; thus, the observed findings are limited. We hope that this study will lead to a large-scale study involving more hospitals and patients that will contribute to advances in the diagnosis and treatment of septic shock. Second, a number of patients were excluded from this study for several reasons and we focused on early septic shock patients in the ED, which might have led to selection bias. Third, the enrollment periods and case volumes varied according to hospital. Institutional characteristics should also be investigated in future studies.

In conclusion, in the KoSS septic shock registry, which included $10 \mathrm{EDs}$, the mortality rate of patients with septic shock exceeded $20 \%$. The results of this preliminary report suggest that there are areas for improvement in terms of the quality of initial resuscitation and the outcomes of patients with septic shock in the ED.

\section{CONFLICT OF INTEREST}

No potential conflict of interest relevant to this article was reported.

\section{ETHICAL DISCLOSURE}

The study was approved by the institutional review boards of the individual participating centers. The institutions and IRB approval numbers are as follows: Asan Medical Center, S2015-1918-0002; Gangnam Sacred Heart Hospital, 2015-11-142; Gangnam Severance Hospital, 3-2015-0227; Hanyang University Hospital, HYUH 2015-11-013-007; Korea University Anam Hospital, HRPC2016184; Korea University Kuro Hospital, KUGH15358-001; Samsung Medical Center, SMC2015-09-057-001; Seoul National University Hospital, J-1408-003-599; Seoul National University Bundang Hospital, B-1409/266-401; Severance Hospital, 4-2015-0929.

\section{REFERENCES}

1. Vincent JL, De Backer D. Circulatory shock. N Engl J Med 2013;369:1726-34.

2. Angus $D C$, van der Poll T. Severe sepsis and septic shock. N Engl J Med 2013;369:840-51.

3. Seymour CW, Rosengart MR. Septic shock: advances in diagnosis and treatment. JAMA 2015;314:708-17.

4. Kaukonen KM, Bailey M, Suzuki S, Pilcher D, Bellomo R. Mortality related to severe sepsis and septic shock among critically ill patients in Australia and New Zealand, 2000-2012. JAMA 2014;311:1308-16.

5. Fleischmann C, Scherag A, Adhikari NK, et al. Assessment of global incidence and mortality of hospital-treated sepsis: current estimates and limitations. Am J Respir Crit Care Med 2016; 193:259-72.

6. Dellinger RP, Levy MM, Rhodes A, et al. Surviving sepsis campaign: international guidelines for management of severe sepsis and septic shock: 2012. Crit Care Med 2013;41:580-637.

7. Vincent JL, Pereira AJ, Gleeson J, Backer D. Early management of sepsis. Clin Exp Emerg Med 2014;1:3-7.

8. ARISE Investigators; ANZICS Clinical Trials Group, Peake SL, et al. Goal-directed resuscitation for patients with early septic shock. N Engl J Med 2014;371:1496-506.

9. ProCESS Investigators, Yealy DM, Kellum JA, et al. A randomized trial of protocol-based care for early septic shock. N Engl J Med 2014;370:1683-93.

10. Mouncey PR, Osborn TM, Power GS, et al. Trial of early, goaldirected resuscitation for septic shock. N Engl J Med 2015; 372:1301-11.

11. Kaukonen KM, Bailey M, Pilcher D, Cooper DJ, Bellomo R. Systemic inflammatory response syndrome criteria in defining severe sepsis. N Engl J Med 2015;372:1629-38.

12. Knaus WA, Draper EA, Wagner DP, Zimmerman JE. APACHE II: a severity of disease classification system. Crit Care Med 1985; 13:818-29.

13. Vincent JL, Moreno R, Takala J, et al. The SOFA (Sepsis-related Organ Failure Assessment) score to describe organ dysfunction/failure. On behalf of the Working Group on Sepsis-Related Problems of the European Society of Intensive Care Medicine. Intensive Care Med 1996;22:707-10.

14. Shankar-Hari M, Phillips GS, Levy ML, et al. Developing a new definition and assessing new clinical criteria for septic shock: for the third international consensus definitions for sepsis and septic shock (Sepsis-3). JAMA 2016;315:775-87.

15. Kadri SS, Rhee $C$, Strich JR, et al. Estimating ten-year trends in septic shock incidence and mortality in United States Aca- 
demic Medical Centers using clinical data. Chest 2017;151: 278-85.

16. Rhodes A, Phillips G, Beale R, et al. The Surviving Sepsis Campaign bundles and outcome: results from the International Multicentre Prevalence Study on Sepsis (the IMPreSS study). Intensive Care Med 2015;41:1620-8.

17. Liu VX, Morehouse JW, Marelich GP, et al. Multicenter implementation of a treatment bundle for patients with sepsis and intermediate lactate values. Am J Respir Crit Care Med 2016; 193:1264-70.
18. Scheer CS, Fuchs $C$, Kuhn SO, et al. Quality improvement initiative for severe sepsis and septic shock reduces 90-day mortality: a 7.5-year observational study. Crit Care Med 2017; 45:241-52.

19. Singer M, Deutschman CS, Seymour CW, et al. The third international consensus definitions for sepsis and septic shock (Sepsis-3). JAMA 2016;315:801-10.

20. Rhodes A, Evans LE, Alhazzani W, et al. Surviving sepsis campaign: international guidelines for management of sepsis and septic shock: 2016. Crit Care Med 2017;45:486-552. 